\title{
A comparison of magnetic overshoots at the bow shocks of Mercury and Saturn
}

\author{
A. Masters, ${ }^{1}$ J. A. Slavin, ${ }^{2}$ G. A. DiBraccio, ${ }^{2}$ T. Sundberg, ${ }^{3}$ R. M. Winslow, ${ }^{4}$ \\ C. L. Johnson, ${ }^{4,5}$ B. J. Anderson, ${ }^{6}$ and H. Korth ${ }^{6}$ \\ Received 15 March 2013; revised 23 May 2013; accepted 30 June 2013; published 25 July 2013.
}

[1] The MErcury Surface, Space ENvironment, GEochemistry, and Ranging (MESSENGER) spacecraft at Mercury and the Cassini spacecraft at Saturn provide us with orbiters around planets at more diverse heliocentric distances than ever before. The dramatically different solar wind conditions at these two planets should mean that Mercury's bow shock is considerably weaker (lower Mach numbers) than Saturn's bow shock. This is expected to produce different magnetic overshoot amplitudes at each bow shock, because the Relative Overshoot Amplitude (ROA) has been shown to increase with both fast magnetosonic Mach number and upstream plasma $\beta$. We qualitatively compare the parameter regimes of Mercury's and Saturn's bow shock by determining ROAs. We analyze 133 MESSENGER encounters with Mercury's bow shock and 90 Cassini encounters with Saturn's bow shock, all with a clear shock ramp. At five of the 133 Mercury bow shock encounters, there is no resolvable magnetic overshoot, whereas all Saturn bow shock encounters have a clear overshoot. We find that the ROA of Mercury's bow shock ranges from $\sim 0$ (no overshoot) to $\sim 0.6$, with a typical value of $\sim 0.2$. We find that the ROA of Saturn's bow shock ranges from $\sim 0.2$ to $\sim 5$, with a typical value of $\sim 2$. This clear ROA difference is consistent with the expected lower fast magnetosonic Mach number and lower upstream plasma $\beta$ at Mercury's bow shock, and we suggest that it is very likely to be primarily caused by the different Mach numbers. This confirmed variation in bow shock parameter regime may produce a different solar wind-magnetosphere interaction at these two planets.

Citation: Masters, A., J. A. Slavin, G. A. DiBraccio, T. Sundberg, R. M. Winslow, C. L. Johnson, B. J. Anderson, and H. Korth (2013), A comparison of magnetic overshoots at the bow shocks of Mercury and Saturn, J. Geophys. Res. Space Physics, 118, 4381-4390, doi:10.1002/jgra.50428.

\section{Introduction}

[2] The interaction between the solar wind and a magnetized planet produces a cavity around the planet, the magnetosphere, within which the planetary magnetic field is essentially confined. Both internal and external plasma conditions vary significantly between the planetary magnetospheres in our solar system, making

\footnotetext{
${ }^{1}$ Institute of Space and Astronautical Science, Japan Aerospace Exploration Agency, Sagamihara, Japan.

${ }^{2}$ Department of Atmospheric Oceanic and Space Sciences, University of Michigan, Ann Arbor, Michigan, USA. USA.

${ }^{3}$ Center for Space Physics, Boston University, Boston, Massachusetts,

${ }^{4}$ Department of Earth Ocean and Atmospheric Sciences, University of British Columbia, Vancouver, British Columbia, Canada.

${ }^{5}$ Planetary Science Institute, Tucson, Arizona, USA.

${ }^{6}$ The Johns Hopkins University Applied Physics Laboratory, Laurel, Maryland, USA.

Corresponding author: A. Masters, Institute of Space and Astronautical Science, Japan Aerospace Exploration Agency, 3-1-1 Yoshinodai, Chuoku, Sagamihara, Kanagawa 252-5210, Japan. (a.masters@stp.isas.jaxa.jp)

(C)2013. American Geophysical Union. All Rights Reserved. 2169-9380/13/10.1002/jgra.50428
}

comparative magnetospheric studies important for understanding how different factors control energy flow through these complex systems.

[3] Data taken by the MErcury Surface, Space ENvironment, GEochemistry, and Ranging (MESSENGER) spacecraft during its orbital tour of Mercury, and data taken by the Cassini spacecraft during its orbital tour of Saturn, allow us to compare the magnetospheres of these two planets (see the reviews by Fujimoto et al. [2007], Slavin et al. [2007], Gombosi et al. [2009], and Mitchell et al. [2009], and references therein). MESSENGER and Cassini are the planetary orbiters located closest to, and furthest from, the Sun to date. Solar wind conditions vary significantly between Mercury and Saturn, allowing us to test our understanding of the solar wind-magnetosphere interaction in different parameter regimes.

[4] Applying scaling laws to solar wind parameters measured in near-Earth space provides predictions of how different the typical solar wind conditions are [Slavin and Holzer, 1981; Russell et al., 1982, 1990; Fujimoto et al., 2007]. A Mach number is defined as the flow speed divided by the speed of a fundamental wave mode (e.g., fast magnetosonic waves), and all such solar wind Mach numbers should increase with heliocentric distance. The ratio of plasma to magnetic pressure 


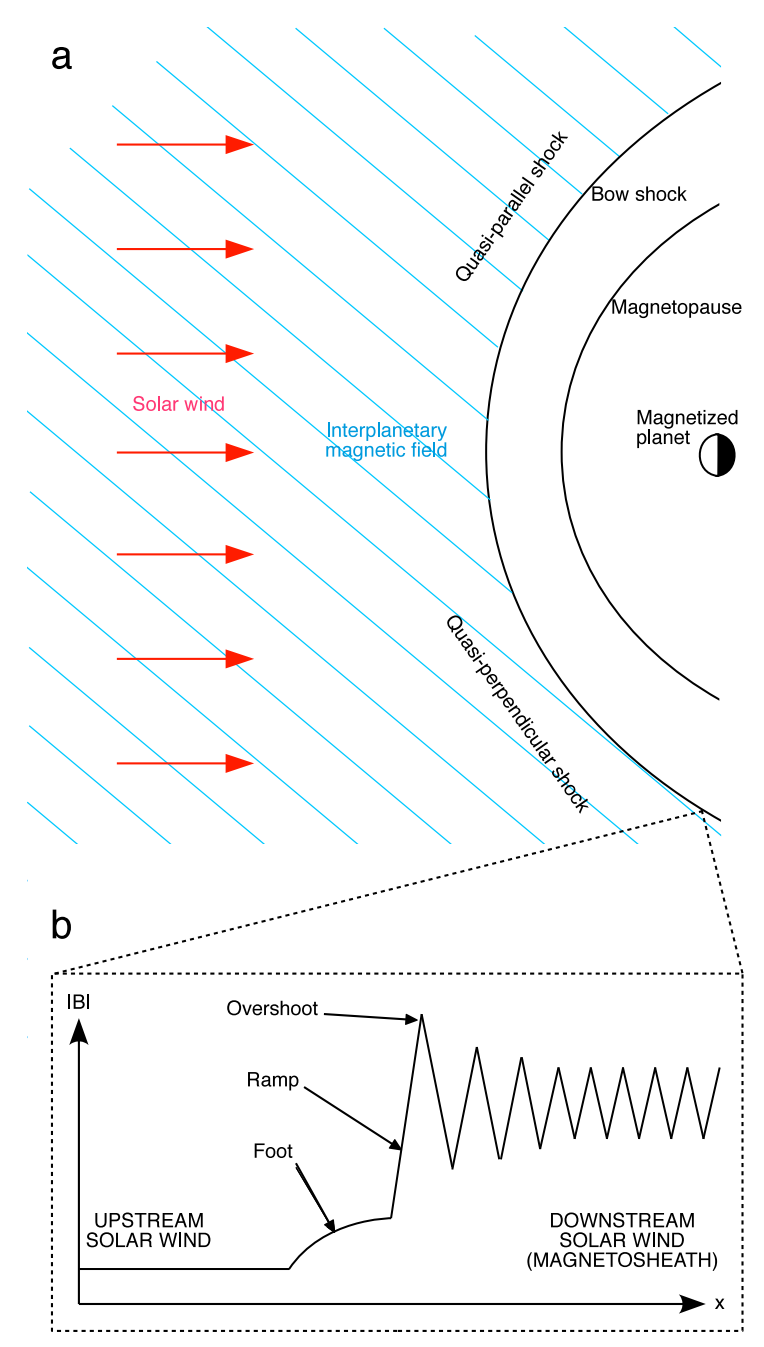

Figure 1. Magnetic structure of a supercritical, quasiperpendicular planetary bow shock. (a) Diagram illustrating the bow shock wave resulting from the interaction between the solar wind and a planetary magnetopause obstacle. An example interplanetary magnetic field orientation is shown upstream of the shock only. Quasi-parallel and quasi-perpendicular regions of the shock surface are indicated. (b) Spatial profile of magnetic field strength across a quasi-perpendicular region of the shock surface. Features of the shock structure are labeled, including the magnetic overshoot. These diagrams are highly simplified, only illustrating relevant features.

(the plasma $\beta$ ) in the solar wind is predicted to increase with heliocentric distance until $\sim 1.5$ Astronomical Units (AU, the mean Earth-Sun distance), before decreasing.

[5] Mercury's heliocentric distance varies between $\sim 0.31$ and $\sim 0.47 \mathrm{AU}$ during an orbit of the Sun, whereas Saturn's heliocentric distance varies between $\sim 9.0$ and $\sim 10.1 \mathrm{AU}$. The expected sonic, Alfvénic, and fast magnetosonic Mach numbers at Mercury are $\sim 6, \sim 5$, and $\sim 4$, respectively (with a variation of $\sim 1$ in all values during a Mercury year), with a plasma $\beta$ of between $\sim 0.5$ and $\sim 0.9$. The expected sonic, Alfvénic, and fast magnetosonic Mach numbers at Saturn are $\sim 12, \sim 13$, and $\sim 10$, respectively, with a plasma $\beta$ of $\sim 1.3$ [Slavin and Holzer, 1981; Russell et al., 1982, 1990; Fujimoto et al., 2007].
[6] As a result, the bow shock wave that stands in the solar wind in front of Mercury's magnetosphere is expected to be significantly weaker (lower Mach numbers) than the equivalent bow shock wave in front of Saturn's magnetosphere, with a more modest difference in upstream plasma $\beta$ between the two shocks. The different bow shock Mach numbers may be primarily responsible for different solar wind-magnetosphere interactions via magnetic reconnection at these planets [Slavin and Holzer, 1979; Scurry and Russell, 1991; Scurry et al., 1994a; Mozer and Hull, 2010]. Plasma $\beta$ conditions in the solar wind downstream of the planetary bow shock (the planetary magnetosheath) are expected to be lower at Mercury compared to at Saturn, due to Mercury's weaker shock. This magnetosheath solar wind encounters the magnetopause boundary of the magnetosphere, where lower plasma $\beta$ not only increases the rate of magnetic reconnection at the magnetopause, but also generally produces more favorable conditions for the onset of reconnection itself [Slavin and Holzer, 1979; Quest and Coroniti, 1981; Paschmann et al., 1986; Scurry and Russell, 1991; Scurry et al., 1994a, 1994b; Swisdak et al., 2003, 2010; Trenchi et al., 2008; Mozer and Hull, 2010; Phan et al., 2010, 2013; Masters et al., 2012; DiBraccio et al., 2013].

[7] MESSENGER observations at Mercury's magnetopause and Cassini observations at Saturn's magnetopause provide support for the predicted difference in solar wind interactions. At Mercury's magnetopause, spacecraft encounters with the reconnection-related phenomenon of Flux Transfer Events (FTEs) are common [Slavin et al., 2008, 2009, 2010, 2012], and the reconnection rate is typically higher than that measured at the terrestrial magnetopause [Slavin et al., 2009; DiBraccio et al., 2013]. In comparison, at Saturn's magnetopause, although Cassini has revealed some evidence for magnetopause reconnection [McAndrews et al., 2008; Lai et al., 2012; Badman et al., 2013], no clear examples of FTEs have been identified to date [Lai et al., 2012], and neither Saturn's magnetopause boundary layer nor Saturn's total auroral power responds strongly to the orientation of the Interplanetary Magnetic Field (IMF) (unlike in the case of Earth's primarily magnetopause reconnection-driven magnetosphere) [Crary et al., 2005; Clarke et al., 2009; Masters et al., 2011a].

[8] Comparing bow shock parameters at Mercury and Saturn with MESSENGER and Cassini data is required to test both our understanding of solar wind evolution with heliocentric distance, and the related explanation of observed differences in magnetopause reconnection at these two planets. However, both the MESSENGER and Cassini spacecraft are three axis stabilized, with limited instrument fields of view that generally prevent the calculation of shock Mach numbers and plasma $\beta$, although limited measurements and estimates based on Cassini data support the expected parameters at Saturn's bow shock [Achilleos et al., 2006; Masters et al., 2011b]. Nonetheless, we can qualitatively compare parameter regimes at the bow shocks of Mercury and Saturn using only magnetic field data, as described below.

[9] The shock angle $\left(\theta_{B n}\right)$ is defined as the angle between the upstream magnetic field and the shock surface normal. Shocks with $\theta_{B n}<45^{\circ}$ are referred to as quasi-parallel, whereas those with $\theta_{B n}>45^{\circ}$ are referred to as quasi-perpendicular. As illustrated in Figure 1, the magnetic structure of a 
quasi-perpendicular shock generally includes an abrupt change in magnetic field strength, referred to as the shock ramp (see the review by Bale et al. [2005] and references therein). Ion reflection and gyration at the shock become a more important energy dissipation mechanism above a critical Mach number, producing both shock foot and overshoot features (Figure 1) (see the reviews by Gosling and Robson [1985] and Gedalin [1997], and references therein). An overshoot is a local maximum in magnetic field strength immediately downstream of the shock ramp (exceeding the downstream field strength), with a spatial scale of a few ion gyroradii [Heppner et al., 1967; Russell and Greenstadt, 1979; Bame et al., 1979; Livesey et al., 1982; Russell et al., 1982; Tatrallyay et al., 1984, 1997; Bagenal et al., 1987; Mellott and Livesey, 1987]. Many previous studies have shown that the relative amplitude of the overshoot positively correlates with both the fast magnetosonic Mach number and the upstream plasma $\beta$ [e.g., Russell et al., 1982].

[10] In this paper, we compare magnetic overshoot features at the bow shocks of Mercury and Saturn. We find significantly smaller overshoots at Mercury's bow shock compared to Saturn's, which is consistent with the expected parameter differences between these planetary bow shocks.

\section{MESSENGER Magnetometer Observations of Mercury's Bow Shock}

[11] We use data taken by the MESSENGER magnetometer, which samples the local magnetic field at a typical rate of 20 vectors per second [Anderson et al., 2007]. All MESSENGER magnetic field data are displayed in Mercury Solar Magnetic (MSM) coordinates, with the origin at the center of Mercury's principally dipolar planetary magnetic field, offset $479 \pm 6 \mathrm{~km}(\sim 0.2$ Mercury radii) northward of the hermographic equator and lying along the planet's spin axis (which is aligned with the magnetic dipole axis) [Anderson et al., 2012]. The $x$ axis points toward the Sun, the $z$ axis points northward and is chosen such that the $x z$ plane contains Mercury's magnetic dipole axis, and the $y$ axis completes the right-handed orthogonal set (directed duskward, in the opposite sense to planetary orbital motion). The distance unit used is Mercury radii $\left(\mathrm{R}_{\mathrm{M}} ; 1 \mathrm{R}_{\mathrm{M}}=2440 \mathrm{~km}\right)$.

[12] MESSENGER's orbital tour of Mercury began on 18 March 2011 and comprises inclined, highly eccentric orbits [Solomon et al., 2007]. During each 88 day long Mercury year, the points of orbit periapsis cover all local time sectors, with the interval during which periapses were within $\pm 2 \mathrm{~h}$ Mercury local time of noon referred to as a "hot season." Figure 2 shows the spacecraft trajectory during example "hot season" orbits. During any orbit the bow shock is encountered in two different regions, one at lower $\left(<90^{\circ}\right)$ and the other at higher $\left(>90^{\circ}\right)$ Solar Zenith Angle (SZA, the angle between the position vector of a crossing and the $x$ axis). Multiple crossings in a region on a single orbit result from shock motion faster than the spacecraft.

[13] The Mach numbers of Mercury's bow shock vary across the shock surface due to changes in the component of the upstream flow vector in the shock normal direction. The highest Mach numbers correspond to the lower SZA region encountered during a "hot season," whereas global modeling of Mercury's bow shock suggests that the Mach
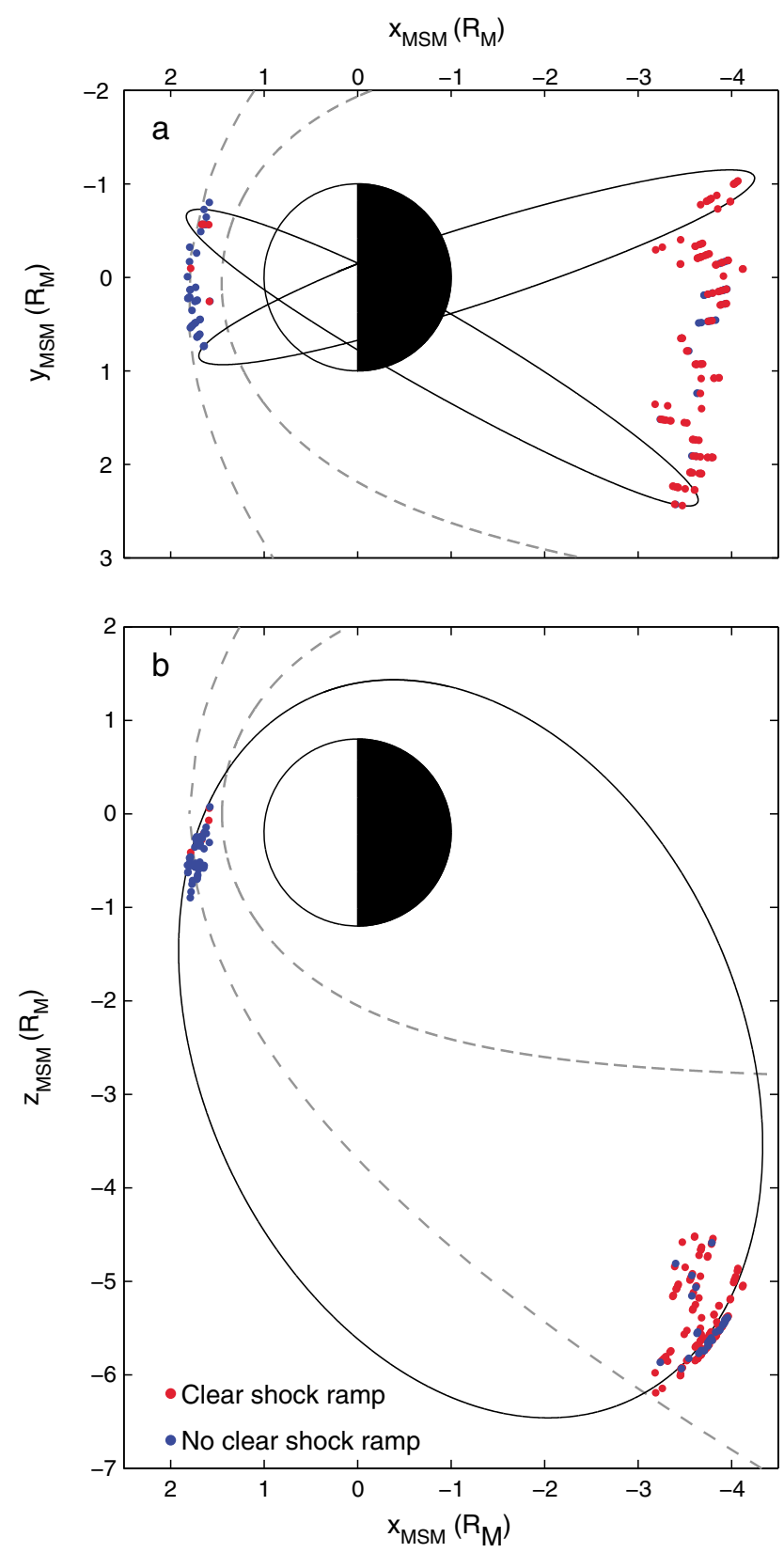

Figure 2. Positions of MESSENGER spacecraft crossings of Mercury's bow shock made during the second "hot season" (20 through 30 August 2011), shown in Mercury Solar Magnetic (MSM) coordinates. (a) Positions projected onto the $x y$ plane. The spacecraft trajectory for the first and last orbits of the "hot season" is shown in black. (b) Positions projected onto the $x z$ plane. The spacecraft trajectory for an orbit in approximately the middle of the "hot season" is shown in black. In both panels, the typical positions of Mercury's bow shock and magnetopause are shown as dashed gray curves, further from and closer to the planet, respectively [Winslow et al., 2013]. Note that although the high-solar zenith angle crossings shown in panel $b$ appear to all have taken place inside the model shock surface, this is an effect of projecting onto the $x z$ plane. The color of the data points indicates whether the shock ramp was clear in the magnetic field data taken during the crossing. 

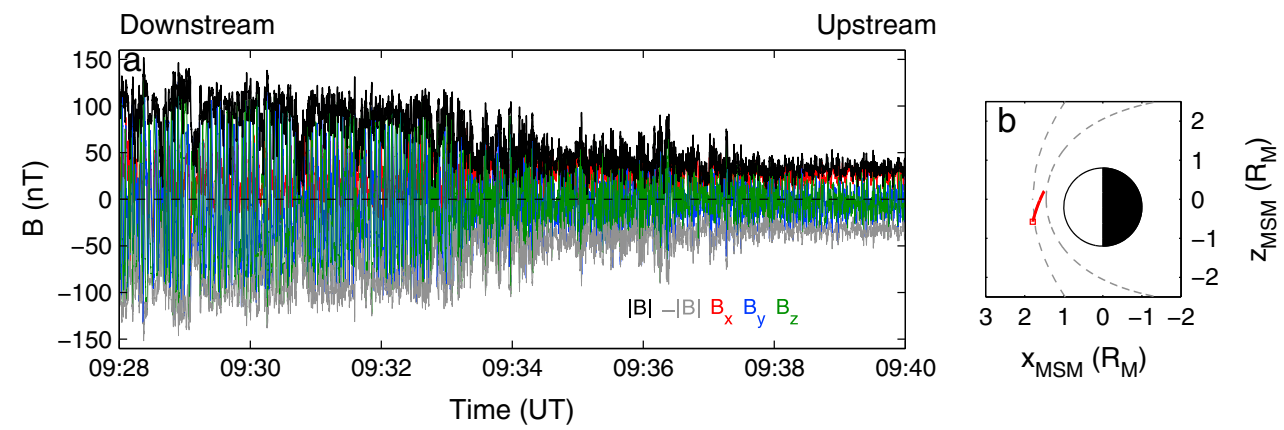

Figure 3. MESSENGER magnetic field observations made on 28 August 2011 when the spacecraft crossed Mercury's subsolar bow shock. (a) Magnetic field data taken between 09:28 and 09:40 Universal Time (UT). (b) Spacecraft trajectory during the time series projected onto the MSM $x z$ plane, given by a red curve. The red square indicates the spacecraft position at the end of the time series. The typical positions of Mercury's bow shock and magnetopause are shown as dashed gray curves, further from and closer to the planet, respectively [Winslow et al., 2013].

numbers in the higher SZA region are $\sim 50 \%$ lower (see Figure 2) [Winslow et al., 2013]. The prevailing (Parker spiral) IMF orientation at Mercury (spiral angle: $\sim 20^{\circ}$ ) means that the lower SZA region is generally quasi-parallel, producing broader magnetic transitions without the clear shock ramp required for our overshoot assessment (see the review by Burgess et al. [2005] and references therein), whereas the higher SZA region is generally quasi-perpendicular, with the clear shock ramp required for our analysis.

[14] We analyzed MESSENGER bow shock crossings made during the second "hot season" (20 through 30 August 2011). This was the first such season when the MESSENGER magnetometer was continuously taking data. Multiple orbits during the season provide many crossings of Mercury's lower SZA subsolar bow shock (highest Mach numbers, but less likely to be quasi-perpendicular) and the higher SZA flank bow shock (lower Mach numbers, but more likely to be quasi-perpendicular).

[15] Magnetic field data were inspected to identify all clear shock crossings, indicated by transitions between a clearly weaker and less variable upstream field, and a clearly stronger and more variable downstream field. Figure 2 shows all identified shock crossings (206 in total). Figure 3 shows the magnetic signature of a typical pass through the shock at lower SZA, which took place on 28 August 2011. The spacecraft began in the magnetosheath solar wind downstream of the shock and ended upstream. The absence of a clear shock ramp and the high level of upstream field fluctuations suggest that this region of the shock was quasi-parallel at this time. Figure 4 shows a typical pass through the shock at higher SZA, which took place on 26 August 2011. The spacecraft began in the solar wind upstream of the shock and ended downstream. Multiple crossings with clear shock ramps took place, with generally lower magnitude upstream field fluctuations, which is characteristic of quasi-perpendicular shocks.

[16] 133 of the 206 identified crossings had clear shock ramps and were included in our overshoot assessment (see section 4). Figure 1 separates those crossings with and those without a clear ramp. Most lower SZA crossings
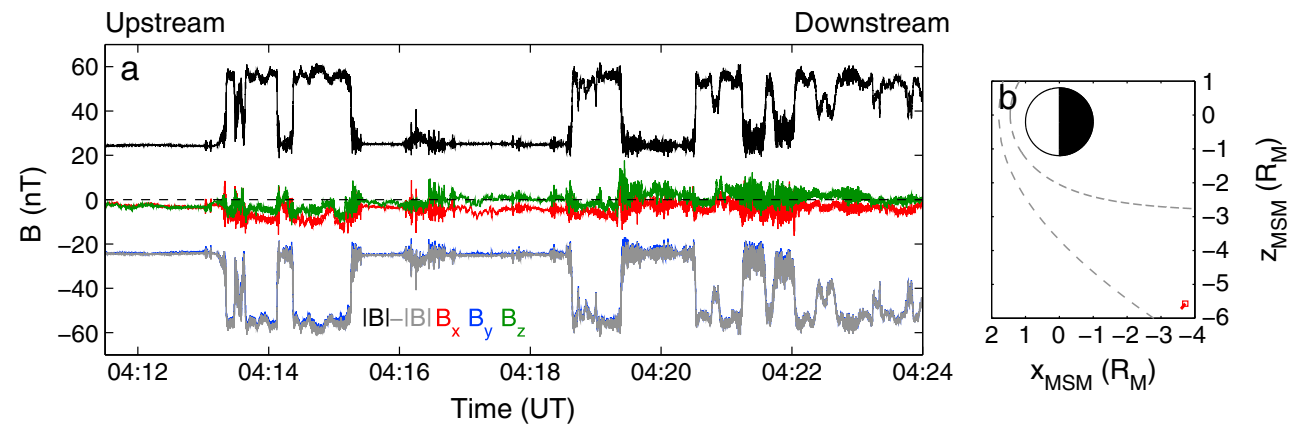

Figure 4. MESSENGER magnetic field observations made on 26 August 2011 when the spacecraft crossed Mercury's flank bow shock. (a) Magnetic field data taken between 04:11:30 and 04:24:00 UT. (b) Spacecraft trajectory during the time series projected onto the MSM $x z$ plane, given by a red curve. The red square indicates the spacecraft position at the end of the time series. The typical positions of Mercury's bow shock and magnetopause are shown as dashed gray curves, further from and closer to the planet, respectively [Winslow et al., 2013]. Note that although panel $b$ appears to show that these high-solar zenith angle crossings occurred inside the model shock surface, this is an effect of projecting onto the $x z$ plane. 

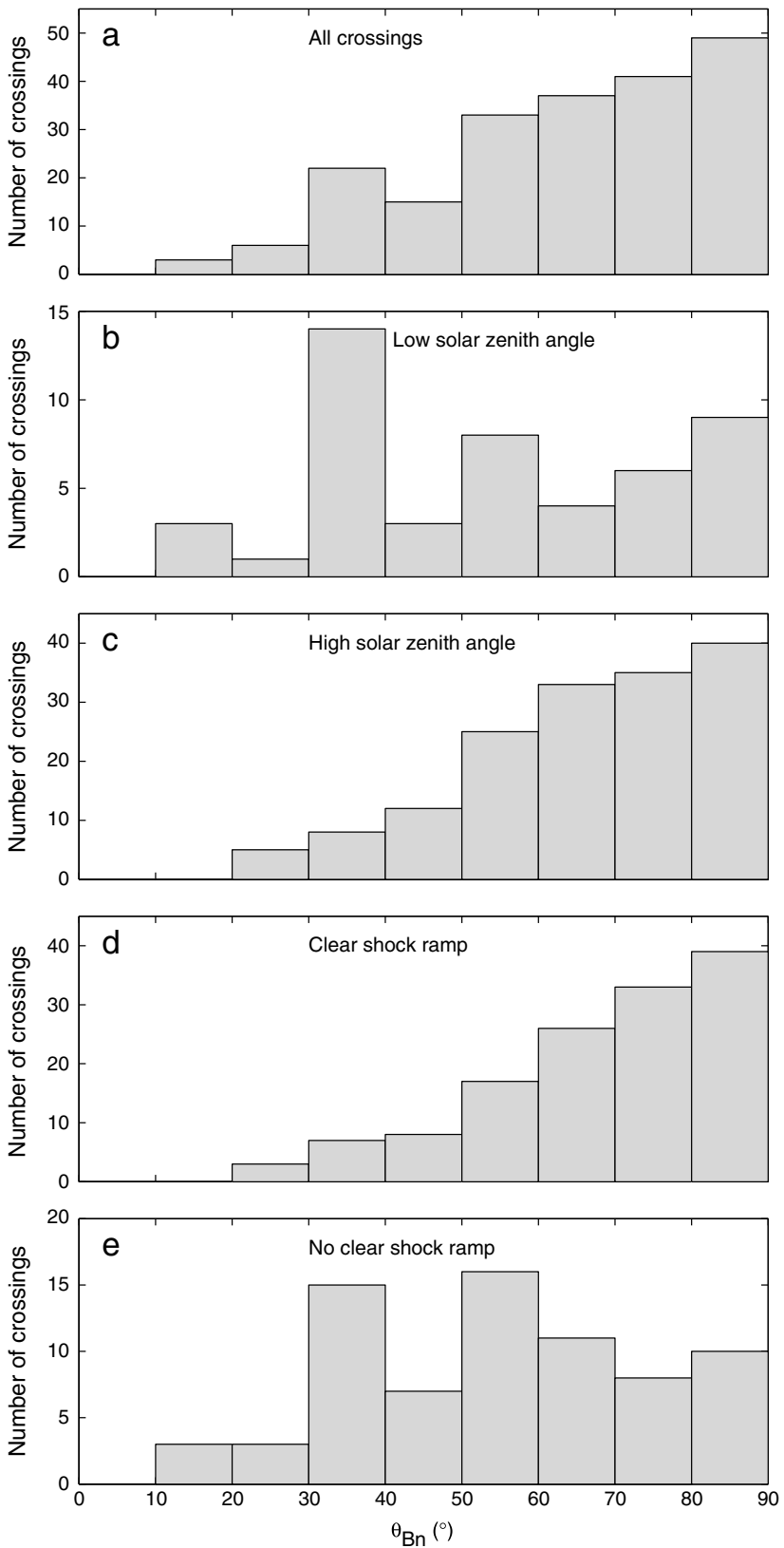

Figure 5. Histograms of the shock angles $\left(\theta_{B n}\right)$ estimated by MESSENGER at Mercury's bow shock between 20 and 30 August 2011. (a) All crossings. (b) Low-solar zenith angle crossings only. (c) High-solar zenith angle crossings only. (d) Crossings with a clear shock ramp only. (e) Crossings without a clear shock ramp only.

did not have a clear ramp and most higher SZA crossings did have a clear ramp, as expected. However, variable IMF conditions produced a number of exceptions to this.

[17] An interval of between $10 \mathrm{~s}$ and $1 \mathrm{~min}$ was selected upstream of all 206 crossings, and the mean field in such intervals was taken as the upstream magnetic field. Combining these upstream fields with a shock surface normal given by a global shape model [Winslow et al., 2013] leads to estimated values of $\theta_{B n}$, shown in histogram format in Figure 5. Crossings with a clear ramp were easier to unambiguously identify (i.e., multiple crossings may have
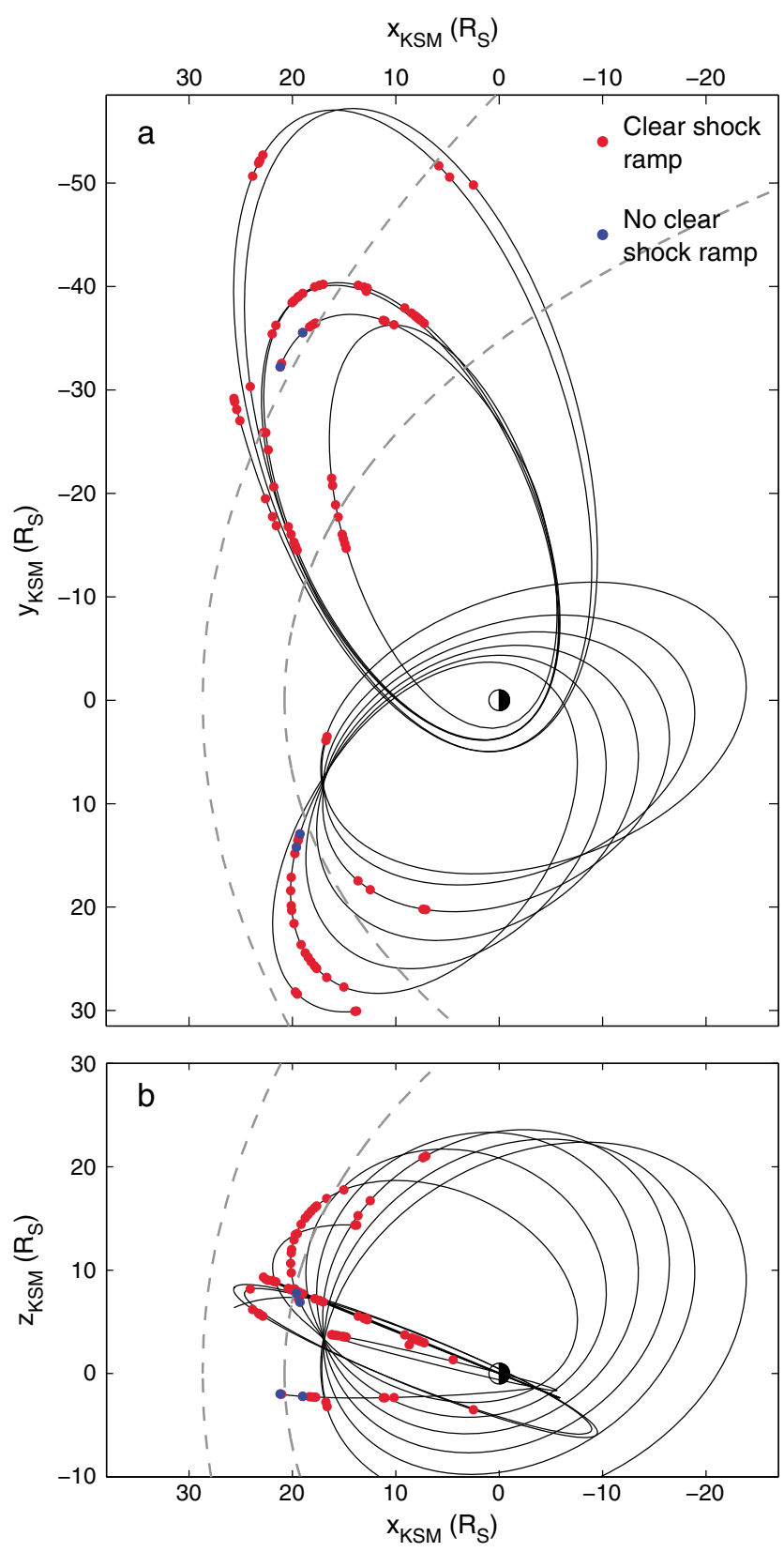

Figure 6. Positions of Cassini spacecraft crossings of Saturn's bow shock made in 2005 and 2007, previously reported by Masters et al. [2011b]. Positions are shown in Kronocentric Solar Magnetospheric (KSM) coordinates. (a) Positions projected onto the $x y$ plane. All crossings that took place at a negative $y$ coordinate occurred in 2005, and all that took place at a positive $y$ coordinate occurred in 2007. (b) Positions projected onto the $x z$ plane. In both panels, the spacecraft trajectory during the orbits where the identified crossings were made is shown in black. Typical positions of Saturn's bow shock and magnetopause are shown as dashed gray curves, further from and closer to the planet respectively [Kanani et al., 2010; Went et al., 2011]. The color of the data points indicates whether the shock ramp was clear in the magnetic field data taken during the crossing. 


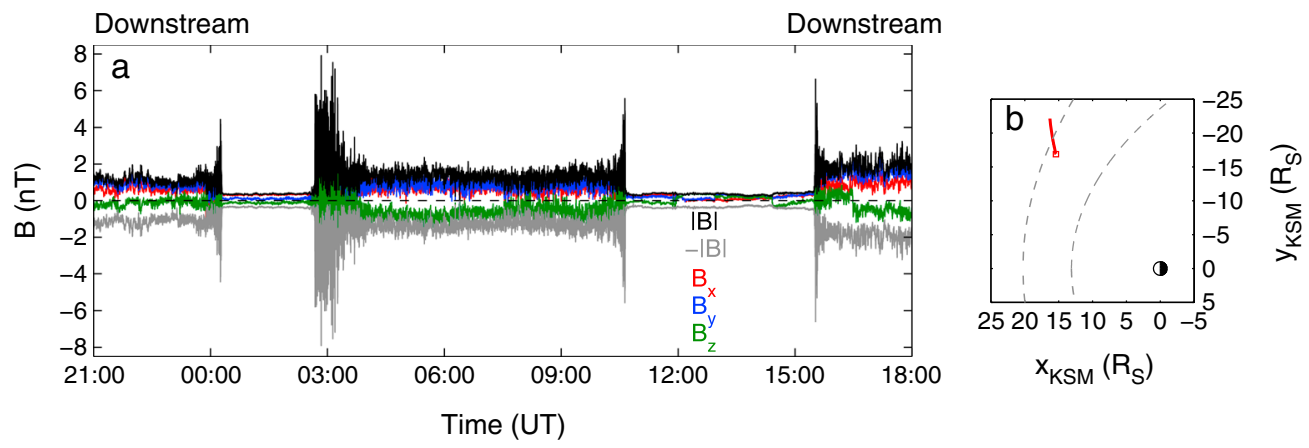

Figure 7. Cassini magnetic field observations made on 11 and 12 April 2005 when the spacecraft crossed Saturn's bow shock. (a) Magnetic field data taken between 21:00 UT on 11 April and 18:00 UT on 12 April. (b) Spacecraft trajectory during the time series projected onto the KSM $x y$ plane, given by a red curve. The red square indicates the spacecraft position at the end of the time series. The typical positions of Saturn's bow shock and magnetopause are shown as dashed gray curves, further from and closer to the planet, respectively [Kanani et al., 2010; Went et al., 2011].

taken place during the interval shown in Figure 3, but cannot be clearly separated), which is partly responsible for the bias towards quasi-perpendicular shock identification (Figure 5a). The combination of faster motion of the flank shock and slower spacecraft motion may have also lead to a greater number of flank (mainly quasi-perpendicular) shock encounters. Separating crossings at low SZA (Figure 5b) or all those without a clear shock ramp (Figure 5e) leads to broader distributions with more values below $45^{\circ}$, likely due to predominantly quasi-parallel shock encounters with significant upstream field fluctuations.

\section{Cassini Magnetometer Observations of Saturn's Bow Shock}

[18] We use data taken by the Cassini fluxgate magnetometer [Dougherty et al., 2004], which samples the local magnetic field at a typical rate of 32 vectors per second. All Cassini magnetic field data are displayed in Kronocentric Solar Magnetospheric (KSM) coordinates (the equivalent of the MSM system used for Mercury in Section 2). The KSM system is Saturn centered, with the positive $x$ axis pointing toward the Sun. The $z$ axis is chosen such that the $x z$ plane contains Saturn's magnetic dipole axis, with the positive $z$ axis pointing northward. The $y$ axis completes the righthanded orthogonal set, directed duskward (in the opposite sense to planetary orbital motion). The unit of distance is Saturn radii $\left(\mathrm{R}_{\mathrm{S}} ; 1 \mathrm{R}_{\mathrm{S}}=60,268 \mathrm{~km}\right)$.

[19] Cassini's orbital tour of Saturn began in July 2004, and hundreds of bow shock crossings have been identified. The vast majority of Cassini bow shock crossings to date have occurred on the dayside, but with few crossings in the subsolar region. Prevailing (Parker spiral) IMF conditions at Saturn (spiral angle: $\sim 84^{\circ}$ ) make the entire dayside shock generally quasi-perpendicular. Here we use the data set reported by Masters et al. [2011b] as the basis of our overshoot assessment for Saturn's bow shock. These authors analyzed a subset of 94 Cassini shock crossings, selected on the basis that more reliable shock parameters could be determined. Figure 6 shows the spacecraft trajectory during the orbits when these crossings occurred, as well as the locations of all 94 crossings. Significant expansion and contraction of the shock surface at speeds of order $100 \mathrm{~km} \mathrm{~s}^{-1}$ [Achilleos et al., 2006] are responsible for the multiple crossings over a large region of space. This coverage of the shock surface roughly illustrates the coverage provided by the entire orbital tour to date.

[20] Note that the SZA distribution of these Cassini crossings differs from that of the MESSENGER crossings of Mercury's bow shock included in this study, which fall into two categories: Low (close to 0) and high SZA (see section 2). These Cassini crossings of Saturn's bow shock cover a single, broader range of SZA, between extremes of 16 and $87^{\circ}$ (see Figure 6). A global model of the shock surface [Went et al., 2011] suggests that the shock Mach numbers should be between $\sim 0$ and $\sim 35 \%$ lower than the values at $\mathrm{SZA}=0^{\circ}$ between these limits of SZA coverage, respectively. The mean Mach number reduction is expected to be $\sim 20 \%$.

[21] Figure 7 shows Cassini magnetometer observations made during a typical set of Cassini crossings of Saturn's bow shock, which took place on 11 and 12 April 2005. The spacecraft began and ended the interval in the magnetosheath solar wind downstream of the shock, making two excursions into the upstream solar wind, each bounded by two shock crossings. The steady upstream field and presence of clear shock ramps suggest that these are quasi-perpendicular crossings. Strong overshoot features are apparent in this time series (see section 4). The magnetic signatures of all 94 Cassini crossings were generally similar to the examples shown in Figure 7.

[22] Masters et al. [2011b] presented a histogram of $\theta_{B n}$ for this set of 94 crossings, showing that few crossings had a value of $\theta_{B n}<45^{\circ}$, as anticipated. We selected all the shock crossings with clear shock ramps for inclusion in our overshoot assessment. Crossings with and without a clear ramp are differentiated in Figure 6, with 90 of the (predominantly quasi-perpendicular) 94 crossings possessing a clear ramp. We limited our analysis to this previously reported set of Cassini shock crossings because 90 crossings with a clear shock ramp is comparable to the 133 clear ramp crossings of Mercury's bow shock resulting from our analysis of MESSENGER data (see section 2). More importantly, as shown in section 4 , this combination of 

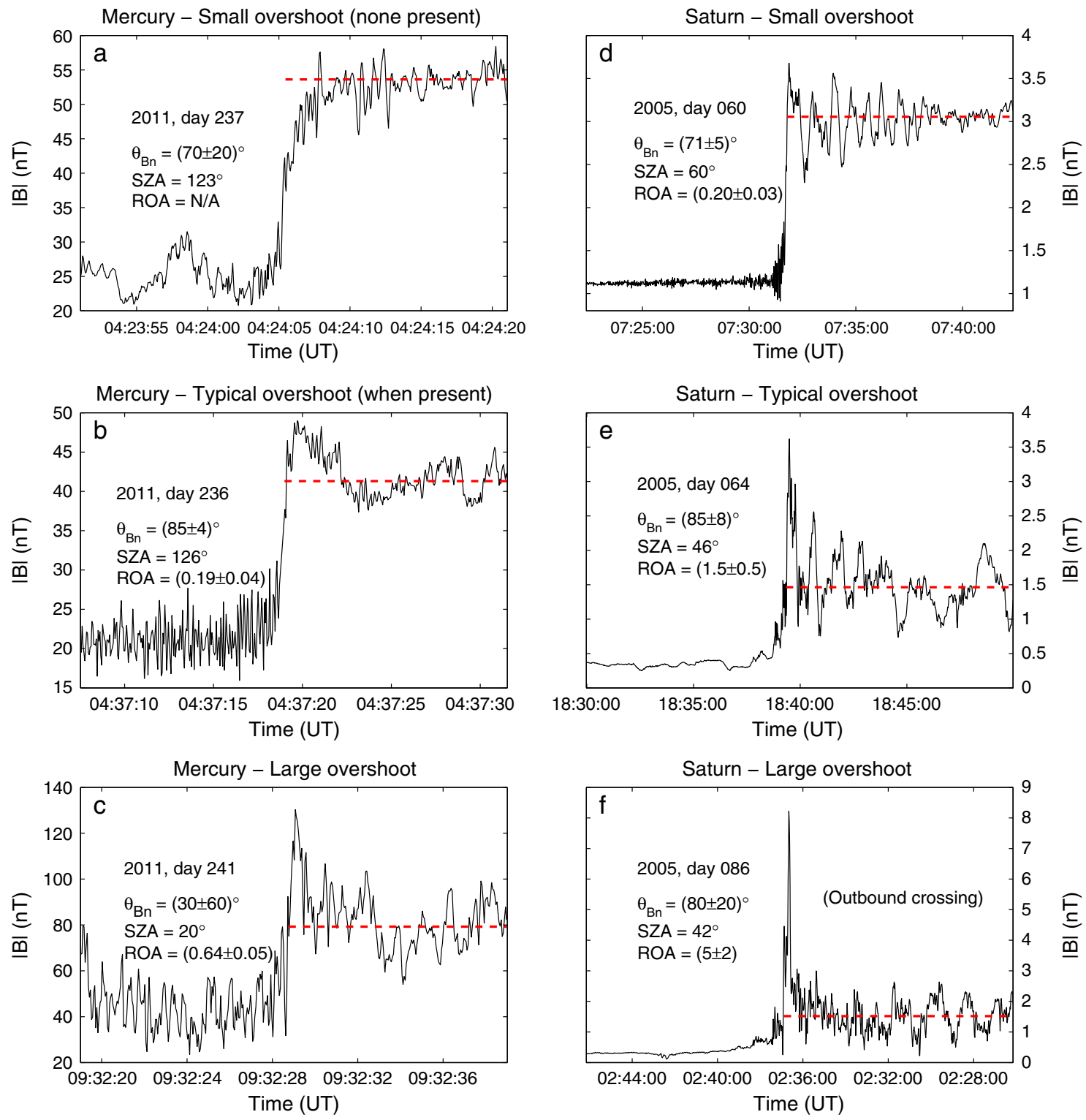

Figure 8. Examples of magnetic signatures of crossings of both Mercury's and Saturn's bow shock. $(a, b, c)$ Magnetic field strength time series measured by MESSENGER during crossings of Mercury's bow shock when a clear shock ramp was observed, showing the smallest, typical, and largest overshoot features, respectively. (d, e, f) Magnetic field strength time series measured by Cassini during crossings of Saturn's bow shock when a clear shock ramp was observed, showing the smallest, typical, and largest overshoot features, respectively. In all panels, the mean downstream field strength is shown as a dashed red line.

planetary bow shock crossing sets is sufficient to reveal a clear difference in the relative amplitude of magnetic overshoot features at the two planetary bow shocks.

\section{Bow Shock Magnetic Overshoot Comparison}

[23] The spatial scale of magnetic overshoots has been shown to be a few upstream convected proton gyroradii [e.g., Livesey et al., 1982]. Assuming 1 upstream convected proton gyroradius, the overshoot spatial scale at Mercury's bow shock is expected to be $\sim 200 \mathrm{~km}$ (B: $\sim 20 \mathrm{nT}$, solar wind speed: $400 \mathrm{~km} \mathrm{~s}^{-1}$ ), whereas at Saturn's bow shock, it should be $\sim 10,000 \mathrm{~km}$ (B: $\sim 0.4 \mathrm{nT}$, solar wind speed: $\sim 400 \mathrm{~km} \mathrm{~s}^{-1}$ ) [e.g., Achilleos et al., 2006]. At both planets, for known spacecraft speeds (a few $\mathrm{km} \mathrm{s}^{-1}$ ) and considering likely upper limit shock speeds of order $100 \mathrm{~km} \mathrm{~s}^{-1}$, we still expect to resolve any overshoot features, given data cadences (see sections 2 and 3 ).

[24] Examples of magnetic overshoot features at the bow shocks of both Mercury and Saturn are shown in Figure 8. To quantify the overshoot, we followed the approach used in previous studies to determine a Relative Overshoot Amplitude (ROA) [e.g., Russell et al., 1982]. For each shock crossing with a clear ramp (both planets), we selected a downstream interval that was sufficiently long (typically $\sim 30 \mathrm{~s}$ and $\sim 5 \mathrm{~min}$ duration for Mercury and Saturn crossings, respectively) to capture the mean downstream magnetic field strength. The interval was chosen to be far 

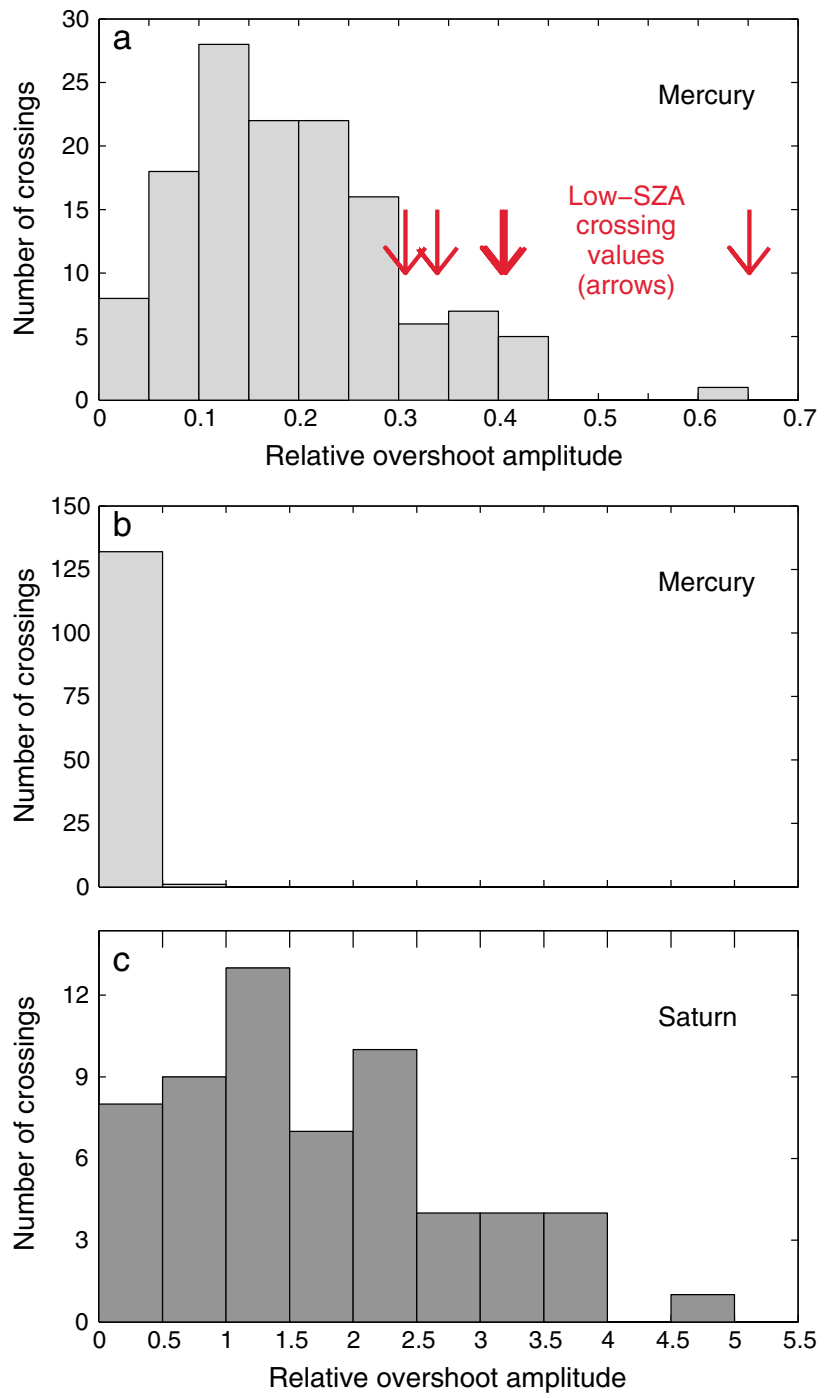

Figure 9. Histograms of relative overshoot amplitude: Comparison of the bow shocks of Mercury and Saturn. (a) All included MESSENGER crossings of Mercury's bow shock with a clear shock ramp. (b, c) All included MESSENGER crossings of Mercury's bow shock and all included Cassini crossings of Saturn's bow shock with clear shock ramps, respectively (using the same histogram bins).

enough downstream to avoid any possible overshoot feature, but close enough to the shock ramp to give a better indication of the downstream field strength at the time of the shock crossing (and possible overshoot encounter). The average field strength in the interval was taken as the downstream field strength, $B_{d}$. All field strength measurements between the ramp and the beginning/end of the chosen downstream interval were isolated, and the maximum value was taken as the overshoot magnetic field strength, $B_{o}$. If this value did not exceed the addition of the standard deviation of the downstream field strengths and $B_{d}$, then the shock was identified as having no clear overshoot feature. If it did exceed it, the ROA was determined as $\left(B_{o}-B_{d}\right) / B_{d}$.

[25] All examples shown in Figure 8 are inbound shock crossings (upstream to downstream), with the exception of the crossing shown in Figure $8 \mathrm{f}$ which is an outbound crossing (downstream to upstream, the time series has been reversed for comparative purposes). Figures $8 \mathrm{a}$ through $8 \mathrm{c}$ show MESSENGER crossings of Mercury's bow shock, and Figures 8d through $8 \mathrm{f}$ show Cassini crossings of Saturn's bow shock. Downstream magnetic field strengths are shown as horizontal dashed red lines in all panels of Figure 8 . These examples have been chosen to illustrate the range of overshoot features revealed by our analysis at each planet. Figures $8 \mathrm{a}$ and $8 \mathrm{~d}$ show the weakest evidence for a shock overshoot at Mercury and Saturn, respectively, Figures $8 \mathrm{~b}$ and $8 \mathrm{e}$ show the typical evidence for a shock overshoot at Mercury and Saturn, respectively, and Figures $8 \mathrm{c}$ and $8 \mathrm{f}$ show the strongest evidence for a shock overshoot at Mercury and Saturn, respectively. The weak overshoot example for Mercury is a high-SZA crossing where there is no clear evidence for an overshoot (Figure 8a), whereas the weakest overshoot example for Saturn still possesses a clear overshoot, with an ROA of $0.20 \pm 0.03$ (Figure $8 \mathrm{~d}$ ). Likewise, comparing the typical overshoot examples (Figures $8 \mathrm{~b}$ and $8 \mathrm{e}$ ) indicates a much larger overshoot at Saturn of $1.5 \pm 0.5$ compared to $0.19 \pm 0.04$ at Mercury. The strongest overshoot feature at Mercury with an ROA of $0.64 \pm 0.05$ was associated with one of the low-SZA crossings with a clear ramp (Figure 8c). However, the strongest overshoot feature at Saturn is almost eight times larger, with an ROA of $5 \pm 2$ (Figure 8f).

[26] We determined the time duration of these overshoot features as the difference between the time when the mean downstream field intersected the shock ramp and the time when the field strength had dropped from the overshoot value to the mean downstream field strength plus one standard deviation (see Figure 8 ). This value was highly variable at both planets, but typically $\sim 5 \mathrm{~s}$ in the case of Mercury's bow shock, and $\sim 60 \mathrm{~s}$ in the case of Saturn's bow shock. Assuming overshoot spatial scales given at the beginning of this section, these time durations suggest typical spacecraftshock speeds of $\sim 40$ and $\sim 100 \mathrm{~km} \mathrm{~s}^{-1}$ at Mercury and at Saturn, respectively. At Saturn's bow shock (where overshoot features are generally clearer), consecutive inbound-outbound crossings often clearly have larger-smaller overshoots, probably due to the influence of shock motion on the Mach number [e.g., Achilleos et al., 2006]. However, there is no clear relationship between ROA and crossing direction (inbound/outbound) for either planet, likely because solar wind variations over the entire crossing set produced more significant changes in the ROA of each bow shock.

[27] ROA statistics for both planets are presented as histograms in Figure 9. Figure 9a shows MESSENGER crossings of Mercury's bow shock only (all with clear ramps). There is no resolvable overshoot feature at five of the 133 included Mercury bow shock crossings. The lowSZA crossings correspond to some of the highest ROA values, likely because of higher Mach numbers in this region (see section 2). Figures 9b and 9c show both Mercury's and Saturn's bow shock (clear ramps only), using the same histogram bins. In general, the ROA of Saturn's bow shock appears to be significantly higher than the ROA of Mercury's bow shock, except potentially for a combination of a case of unusually low ROA of Saturn's bow shock and a case of unusually high ROA of Mercury's bow shock. The strongest dayside overshoot examples at Mercury's bow shock are still far weaker than the overshoots of the majority of the Saturn bow shock crossing set. 


\section{Conclusion}

[28] Our analysis has revealed significantly smaller magnetic overshoots at Mercury's bow shock compared to at Saturn's. This is consistent with the expected difference between typical parameters at these two planetary bow shocks, since both the predicted lower fast magnetosonic Mach number and lower upstream plasma $\beta$ at Mercury should reduce the overshoot amplitude (see section 1) [e.g., Russell et al., 1982]. We cannot determine the relative importance of the different Mach number and the different upstream plasma $\beta$, although it is expected that a (more significant) Mach number difference is primarily responsible.

[29] Previous studies that have also compared overshoot amplitude at different planetary bow shocks support that the Mach number variation is more significant. Although these past comparative studies did not consider Mercury's bow shock (or as many crossings of Saturn's bow shock), they demonstrated that while upstream plasma $\beta$ does influence overshoot amplitude, the range of this parameter is roughly similar at Venus, Earth, Jupiter, and Saturn [Russell et al., 1982]. However, ranges of fast magnetosonic Mach number were found to be clearly different and mainly responsible for larger overshoots at the bow shocks of planets at greater heliocentric distances [Russell et al., 1982; Bagenal et al., 1987]. The typical overshoot amplitudes at the bow shocks of Mercury and Saturn reported here $(\sim 0.2$ and $\sim 2$, respectively) are consistent with the relationship between planetary bow shock ROA and fast magnetosonic Mach number revealed by these past studies, based on the expected fast magnetosonic Mach number at these two planets. We thus suggest that the expected significantly lower Mach number of Mercury's bow shock compared to that of Saturn's is very likely the principal factor controlling the difference in magnetic overshoot amplitudes.

[30] Note that even if the difference in overshoot amplitude is instead principally due to lower upstream plasma $\beta$ at Mercury compared to Saturn, rather than a difference in Mach number, the implications for downstream plasma $\beta$ conditions would be the same. In either case, the parameter differences favor lower plasma $\beta$ conditions downstream of Mercury's bow shock compared to downstream of Saturn's. Indeed, as discussed in section 1, these shock parameter differences are thought to affect downstream plasma $\beta$ conditions in this way, resulting in different solar wind-magnetosphere interactions at Mercury and Saturn. However, further assessment of this proposal requires consideration of the influence of shock geometry. As we have seen in the present study, Mercury's dayside bow shock is generally quasi-parallel, whereas Saturn's is generally quasi-perpendicular. This difference in shock geometry (resulting from the prevailing IMF orientation throughout the heliosphere) will also affect downstream $\beta$ conditions and so must be considered carefully in the course of further work on this topic.

[31] Acknowledgments. AM acknowledges the support of the JAXA International Top Young Fellowship Program. We acknowledge the Cassin magnetometer Principal Investigator M.K. Dougherty and the support of the MAG data processing/distribution staff associated with both the MESSENGER and Cassini-Huygens missions. RMW and CLJ acknowledge support from the Natural Sciences and Engineering Research Council of Canada and MESSENGER Participating Scientist grant NNX11AB84G.

[32] Philippa Browning thanks Scott Boardsen and another reviewer for their assistance in evaluating this paper.

\section{References}

Achilleos, N., et al. (2006), Orientation, location and velocity of Saturn's bow shock: Initial results from the Cassini spacecraft, J. Geophys. Res., 111, A03201, doi:10.1029/2005JA011297.

Anderson, B. J., et al. (2007), The Magnetometer instrument on MESSENGER, Space Sci. Rev., 131, 417-450, doi:10.1007/s11214007-9246-7.

Anderson, B. J., C. L. Johnson, H. Korth, R. M. Winslow, J. E. Borovsky, M. E. Purucker, J. A. Slavin, S. C. Solomon, M. T. Zuber, and R. L. McNutt Jr. (2012), Low-degree structure in Mercury's planetary magnetic field, J. Geophys. Res., 117, E00L12, doi:10.1029/2012JE004159.

Badman, S. V., A. Masters, H. Hasegawa, M. Fujimoto, A. Radioti, D. Grodent, N. Sergis, M. K. Dougherty, and A. J. Coates (2013), Bursty magnetic reconnection at Saturn's magnetopause, Geophys. Res. Lett., 40, 1027-1031, doi:10.1002/grl.50199.

Bagenal, F., et al. (1987), The Uranian bow shock: Voyager 2 inbound observations of a high Mach number shock, J. Geophys. Res., 92, 8603-8612.

Bale, S. D., et al. (2005), Quasi-perpendicular shock structure and processes, Space Sci. Rev., 118, 161-203.

Bame, S. J., J. R. Asbridge, J. T. Gosling, M. Halbig, G. Paschmann, N. Sckopke, and H. Rosenbauer (1979), High temporal resolution observations of electron heating at the bow shock, Space Sci. Rev., 23, 75-92.

Burgess, D., et al. (2005), Quasi-parallel shock structure and processes, Space Sci. Rev., 118, 205-222.

Clarke, J. T., et al. (2009), Response of Jupiter's and Saturn's auroral activity to the solar wind, J. Geophys. Res., 114, A05210, doi:10.1029/ 2008JA013694

Crary, F. J., et al. (2005), Solar wind dynamic pressure and electric field as the main factors controlling Saturn's aurorae, Nature, 433, 720-722.

DiBraccio, G. A., J. A. Slavin, S. A. Boardsen, B. J. Anderson, H. Korth, T. H. Zurbuchen, J. M. Raines, D. N. Baker, R. L. McNutt Jr., and S. C. Solomon (2013), MESSENGER observations of magnetopause structure and dynamics at Mercury, J. Geophys. Res. Space Physics, 118 , 1-12, doi: $10.1002 /$ jgra.50123.

Dougherty, M. K., et al. (2004), The Cassini magnetic field investigation, Space Sci. Rev., 114, 331-383.

Fujimoto, M., W. Baumjohann, K. Kabin, R. Nakamura, J. A. Slavin, N. Terada, and L. Zelenyi (2007), Hermean magnetosphere-solar wind interaction, Space Sci. Rev., 132, 529-550.

Gedalin, M. (1997), Ion dynamics and distribution at the quasi-perpendicular collisionless shock front, Surv. Geophys., 18, 541-566.

Gombosi, T. I., et al. (2009), Saturn's magnetospheric configuration, in Saturn From Cassini-Huygens, edited by M. K Dougherty, L. W. Esposito, and S. M. Krimigis, pp. 203-256, Springer, New York.

Gosling, J. T., and A. E. Robson (1985), Ion reflection, gyration, and dissipation at supercritical shocks, in Collisionless Shocks in the Heliosphere: Reviews of Current Research, Geophys. Monogr. Ser., vol. 35, edited by B. T. Tsurutani and R. G. Stone, p. 141, AGU, Washington, D. C.

Heppner, J. P., M. Sugiura, T. L. Skillman, B. G. Ledley, and M. Campbell (1967), OGO-A magnetic field observations, J. Geophys. Res., 72, 5417-5471.

Kanani, S. J., et al. (2010), A new form of Saturn's magnetopause using a dynamic pressure balance model, based on in situ, multi-instrument Cassini measurements, J. Geophys. Res., 115, A06207, doi:10.1029/ 2009JA014262.

Lai, H. R., H. Y. Wei, C. T. Russell, C. S. Arridge, and M. K. Dougherty (2012), Reconnection at the magnetopause of Saturn: Perspective from FTE occurrence and magnetosphere size, J. Geophys. Res., 117, A05222, doi:10.1029/2011JA017263.

Livesey, W. A., C. F. Kennel, and C. T. Russell (1982), ISEE-1 and -2 observations of magnetic field strength overshoots in quasi-perpendicular bow shocks, Geophys. Res. Lett., 9, 1037-1040.

Masters, A., D. G. Mitchell, A. J. Coates, and M. K. Dougherty (2011a), Saturn's low-latitude boundary layer 1: Properties and variability, J. Geophys. Res., 116, A06210, doi:10.1029/2010JA016421.

Masters, A., S. J. Schwartz, E. M. Henley, M. F. Thomsen, B. Zieger, A. J. Coates, N. Achilleos, J. Mitchell, K. C. Hansen, and M. K. Dougherty (2011b), Electron heating at Saturn's bow shock, J. Geophys., 116, A10107, doi:10.1029/2011JA016941

Masters, A., J. P. Eastwood, M. Swisdak, M. F. Thomsen, C. T. Russell, N. Sergis, F. J. Crary, M. K. Dougherty, A. J. Coates, and S. M. Krimigis (2012), The importance of plasma $\beta$ conditions for magnetic reconnection at Saturn's magnetopause, Geophys. Res. Lett., 39, L08103, doi:10.1029/2012GL051372.

McAndrews, H. J., C. J. Owen, M. Thomsen, B. Lavraud, A. Coates, M. Dougherty, and D. T. Young (2008), Evidence for reconnection at Saturn's magnetopause, J. Geophys. Res., 113, A04210, doi:10.1029/ 2007JA012581. 


\section{MASTERS ET AL.: BOW SHOCK OVERSHOOT: MERCURY AND SATURN}

Mellott, M. M., and W. A. Livesey (1987), Shock overshoots revisited, J. Geophys. Res., 92, 13,661-13.665.

Mitchell, D. G., et al. (2009), The dynamics of Saturn's magnetosphere, in Saturn From Cassini-Huygens, edited by M. K Dougherty, L. W. Esposito, and S. M. Krimigis, pp. 257-280, Springer, New York.

Mozer, F. S., and A. Hull (2010), Scaling the energy conversion rate from magnetic field reconnection to different bodies, Phys. Plasmas, 17, 10,2906.

Paschmann, G., I. Papamastorakis, W. Baumjohann, N. Sckopke, C. W. Carlson, B. U. Sonnerup, and H. Lühr (1986), The magnetopause for large magnetic shear: AMPTE/IRM observations, J. Geophys. Res., 91, 11,099-11,115.

Phan, T.-D., et al. (2010), The dependence of magnetic reconnection on plasma $\beta$ and magnetic shear: Evidence from solar wind observations, Astrophys. J. Lett., 719, L199-L203.

Phan, T. D., G. Paschmann, J. T. Gosling, M. Oieroset, M. Fujimoto, J. F. Drake, and V. Angelopoulos (2013), The dependence of magnetic reconnection on plasma $\beta$ and magnetic shear: Evidence from magnetopause observations, Geophys. Res. Lett., 40, 11-16, doi:10.1029/2012GL054528.

Quest, K. B., and F. V. Coroniti (1981), Linear theory of tearing in a high- $\beta$ plasma, J. Geophys. Res., 86, 3299-3305.

Russell, C. T., and E. W. Greenstadt (1979), Initial ISEE magnetometer results: Shock observation, Space Sci. Rev., 23, 3-37.

Russell, C. T., M. M. Hoppe, and W. A. Livesey (1982), Overshoots in planetary bow shocks, Nature, 296, 45-48.

Russell, C. T., R. P. Lepping, and C. W. Smith (1990), Upstream waves at Uranus, J. Geophys. Res., 95, 2273-2279.

Scurry, L., and C. T. Russell (1991), Proxy studies of energy transfer to the magnetosphere, J. Geophys. Res., 96(A6), 9541-9548.

Scurry, L., C. T. Russell, and J. T. Gosling (1994a), Geomagnetic activity and the beta dependence of the dayside reconnection rate, J. Geophys. Res., 99, 14,811-14,814.

Scurry, L., C. T. Russell, and J. T. Gosling (1994b), A statistical study of accelerated flow events at the dayside magnetopause, J. Geophys. Res., 99 14,815-14,829.

Slavin, J. A., and R. E. Holzer (1979), The effect of erosion on the solar wind stand-off distance at Mercury, J. Geophys. Res., 84, 2076-2082.

Slavin, J. A., and R. E. Holzer (1981), Solar wind flow about the terrestrial planets 1. Modeling bow shock position and shape, J. Geophys. Res., 86, 11,401-11,418, doi:10.1029/JA086iA13p11401.
Slavin, J. A., et al. (2007), MESSENGER: Exploring Mercury's magnetosphere, Space Sci. Rev., 131, 133-160.

Slavin, J. A., et al. (2008), Mercury's magnetosphere after MESSENGER's first flyby, Science, 321, 85-89, doi:10.1126/science.1159040.

Slavin, J. A., et al. (2009), MESSENGER observations of magnetic reconnection in Mercury's magnetosphere, Science, 324, 606-610.

Slavin, J. A., et al. (2010), MESSENGER observations of large flux transfer events at Mercury, Geophys. Res. Lett., 37, L02105, doi:10.1029/ 2009GL041485.

Slavin, J. A., et al. (2012), MESSENGER observations of a flux-transferevent shower at Mercury, J. Geophys. Res., 117, A00M06, doi:10.1029/ 2012JA017926.

Solomon, S. C., et al. (2007), MESSENGER mission overview, Space Sci. Rev., 131, 3-39, doi:10.1007/s11214-007-9247-6.

Swisdak, M., B. N. Rogers, J. F. Drake, and M. A. Shay (2003), Diamagnetic suppression of component magnetic reconnection at the magnetopause, J. Geophys. Res., 108(A5), 1218, doi:10.1029/2002JA009726.

Swisdak, M., M. Opher, J. F. Drake, and F. Alouani Bibi (2010), The vector direction of the interstellar magnetic field outside the heliosphere, Astrophys. J., 710, 1769-1775.

Tatrallyay, M., J. G. Luhmann, and C. T. Russell (1984), Magnetic overshoots in the Venus bow shock, Adv. Space Res., 4, 283-286.

Tatrallyay, M., G. Gévai, I. Apáthy, K. Schwingenschuh, T.-L. Zhang, G. A. Kotova, M. I. Verigin, S. Livi, and H. Rosenbauer (1997), Magnetic field overshoots in the Martian bow shock, J. Geophys. Res., 102, 2157-2163.

Trenchi, L., M. F. Marcucci, G. Pallocchia, G. Consolini, M. B. Bavassano Cattaneo, A. M. Di Lellis, H. Rème, L. Kistler, C. M. Carr, and J. B. Cao (2008), Occurrence of reconnection jets at the dayside magnetopause: Double Star observations, J. Geophys. Res., 113 A07S10, doi:10.1029/2007JA012774.

Went, D. R., G. B. Hospodarsky, A. Masters, K. C. Hansen, and M. K. Dougherty (2011), A new semi-empirical model of Saturn's bow shock based on propagated solar wind parameters, J. Geophys. Res., 116, A07202, doi:10.1029/2010JA016349.

Winslow, R. M., B. J. Anderson, C. L. Johnson, J. A. Slavin, H. Korth, M. E. Purucker, D. N. Baker, and S. C. Solomon (2013), Mercury's magnetopause and bow shock from MESSENGER Magnetometer observations, J. Geophys. Res. Space Physics, 118, 2213-2227, doi:10.1002/jgra.50237. 